\title{
Spectrum of acute leukemias diagnosed on flow cytometry: Analysis from tertiary care centre from North India
}

\author{
Surendra Koju, ${ }^{1 *}$ Man Updesh Singh Sachdeva, ${ }^{2}$ Praveen Bose, ${ }^{2}$ Neelam Varma ${ }^{2}$
}

BACKGROUND: Acute leukemias (ALs) are a heterogeneous group of malignancies with varying clinical, morphologic, immunologic, and molecular characteristics. WHO 2008 classification of ALs require a multi-parametric approach to the diagnosis. This study aims to evaluate the role of flow cytometry in diagnosis and sub-classification of acute leukemias.

METHODS: Consecutive patients of adult and paediatric ALs during June 2012 to May 2013 were retrospectively analyzed and studied using BD FACS Canto-II flow cytometer. The results of immunophenotyping were reviewed and analyzed for cross-lineage antigen expression.

RESULT: Over a period of year, 422 individuals were diagnosed as AL. There were 287 males and 135 females with $\mathrm{M}: \mathrm{F}=2 \cdot 1: 1$. There were 237 adults $\&$ 185 children. $36.3 \%$ were AML and $60.4 \%$ were ALL, while $3.3 \%$ of cases were mixed phenotypic acute leukemia (MPAL). The commonest WHO subtype in AML group was AML with maturation being 31\%. In case of ALL there were $83.9 \%$ B-ALLs and $16.1 \% \mathrm{~T}$ ALLs. In MPAL B-Myeloid was 71.4\%, whereas TMyeloid was $28.6 \%$ of cases. Both AML and MPAL were more frequently seen in adults accounting to $83 \%$ and $92.9 \%$ respectively of all ALs cases. In contrast, $62 \%$ of ALLs were children and only $38 \%$ were adults. Out of all ALs, $37.6 \%$ of showed cross lineage antigen expression. In AML, B-ALL and T-ALL cross lineage antigen expression were $26.14 \%, 39.71 \%$ and $82.9 \%$ respectively.

CONCLUSION: Flow cytometry is useful in diagnosis and sub classification of AL. It is essential in cytochemical myeloperoxidase (MPO) negative cases. Cross- lineage antigen expression is frequent in ALs, and hence, lineage specific intra-cytoplasmic antibodies including anti-MPO and cytoplasmic-CD3 are essential for correct categorization of ALs.

Key words: Flow cytometer, Immuophenotyping, Acute leukemia

(C) 2015 Nepalese Association for Clinical Chemistry

\section{Introduction}

Acute leukemias are a heterogeneous group of malignancies with varying clinical, morphologic, immunologic, and molecular characteristics. Flow cytometric immunophenotyping is a valuable tool for the diagnosis, classification, staging, and monitoring of acute leukemia. Differentiation between myeloid and lymphoid leukemias, most often made by flow cytometry, is important [1, 2]. Several advances in flow cytometry, including availability of new monoclonal antibodies, improved gating strategies, and multiparameter analytic techniques, have all dramatically improved the utility of flow cytometry in the diagnosis and classification of leukemia. Detailed understanding of phenotypic patterns of differentiation, particularly in myeloid leukemia, allows for more precise classification of leukemia than does morphology alone [3].

2008 World Health Organization (WHO) classification of hematolymphoid malignancy requires a multiparametric approach to diagnosis and outlines the morphologic, immunophenotypic, and genotypic features characteristic of each disease entity. Many of genetically distinct subgroups of leukemia have been found to be closely associated with distinct immunophenotypes. Thus, in addition to classification into differentiation-based subtypes, detailed flow cytometric studies can define complex antigenic profiles that are associated with specific molecular defects and well-defined biology. In summary, multiparameter flow cytometry is an invaluable tool in the diagnosis, classification, and monitoring of patients with acute leukemia.

The purpose of the study was to evaluate the role of flow cytometry in diagnosis and proper classification of acute leukemias. This study aims to find out the frequency of cross lineage antigen

\footnotetext{
'Department of Pathology, Dhulikhel Hospital-Kathmandu University Hospital, ${ }^{2}$ Department of Hematology,

Postgraduate Institute of Medical Education and Research, Chandigarh, India

Correspondence to: Surendra Koju, Department of Pathology, Dhulikhel Hospital-Kathmandu University Hospital P.O.BoxNo:11008, Kathmandu, Nepal. Phone number: 9841791258, E-mail: suren.koju@gmail.com
} 
expression in AML, B-ALL and T-ALL.

\section{Methods}

Four hundred and twenty two patients of adult and paediatric acute leukemias, diagnosed in the Department of Hematology, PGIMER, Chandigarh, during June 2012 to May 2013 were retrospectively analysed. In addition to routine evaluation by complete blood counts, the patients were evaluated with peripheral blood films, bone marrow aspirate $\&$ trephine biopsy, using May Grunwald Giemsa, Hematoxylin \& Eosin and cytochemical stains. Bone marrow aspirate and/or peripheral blood samples collected from all the patients were processed with standardized "lyse-stain-wash" technique, stained with 4 colors combination of antibody cocktails and acquired on dual laser BD FACS Canto II flow cytometer. All samples were processed within 24 hour of collection.

Immunophenotyping was done in mononuclear cell obtained by lysing whole blood by BD FACS lysing solution. For immunophenotyping various combination of flurochrome conjugated monoclonal antibodies (MoAbs) were added per tube in sample. They were conjugated with fluorescein isothiocyanate (FITC), phycoerythrin (PE), Allophycocyanine (APC) or peridinin chlorophyll protein $(\mathrm{PerCP})$, and were directed to antigen of myeloid cell, B cell, $\mathrm{T}$ cell, monocyte or immature precursor cells. Data were acquired and blast gating strategy included using dot plots of CD45 expression versus intracellular complexity (side scatter angle, SSC) and also a second gate was based on cell forward scatter angle, (FSC) versus SSC.

Demographic profile of the patients, including age, sex was recorded for statistical analysis. Immunophenotypic profiles of all patients were analysed for cross lineage antigen expression.

\section{Results}

Over a period of one year, 422 individuals were diagnosed as acute leukemia. Diagnosis was based on morphology, cytochemistry and immunophenotyping by flow cytometer. There were 287 males and 135 females with $\mathrm{M}: \mathrm{F}=2.1: 1$ (Table $1)$. Fifty six percent $(237 / 422)$ were adults \& $43.8 \%(185 / 422)$ were paediatric cases (Table 2$)$.

In 422 cases of acute leukemia, 36.3\% (153/422) were classified as acute myeloid leukemia (AML) and $60.4 \%(255 / 422)$ were acute lymphoblastic leukemia (ALL), while remaining 3.3\% (14/422) of cases were mixed phenotypic acute leukemia (MPAL). The commonest WHO subtype in AML group was AML with maturation (FABM2) being 31\% (47/153) (Table 3). In case of ALL there were $83.9 \%(214 / 255)$ B-ALLs and $16.1 \%$ (41/255) T-ALLs (Table 4). The most common subtype in MPAL was B-Myeloid, accounting for $71.4 \%(10 / 14)$, whereas TMyeloid were $28.6 \%$ (4/14) of the MPAL cases. There was no case of B-T or tri-lineage MPAL (Table 5). AML was much more frequently seen in adults accounting to $83 \%(127 / 153)$ of all AML cases, and rest $17 \%(26 / 153)$ occurred in children. In contrast, $62 \%(158 / 255)$ of all ALL cases were children and only 38\% (97/255) were adults. Similar to the distribution of AML, $92.9 \%(13 / 14)$ cases of MPAL were adults and only $7.1 \%(1 / 14)$ were children.

Out of 422 cases of acute leukemia 37.6\% (159/422) showed cross lineage antigen expression. Out of 153 cases of AML, 40 cases showed cross lineage antigen expression. B lineage antigens were expressed in $22.5 \%(9 / 40)$, $\mathrm{T}$ lineage markers were seen in $75 \%(30 / 40)$ and both $\mathrm{B} \& \mathrm{~T}$ lineage markers were present in $2.5 \%(1 / 40)$ cases. Similarly, in B-ALL, 85 out of 214 cases showed cross antigen expression comprising of myeloid lineage, T-lineage and combined myeloid-T-lineage cross expression in

Table 1. Frequency of type acute leukemias according to sex

\begin{tabular}{lccc}
\hline Types of acute leukemia & Male (n= 287) & $\begin{array}{c}\text { Female (n=135) } \\
\mathbf{n}(\mathbf{n})\end{array}$ & $\begin{array}{c}\text { Total (n=422) } \\
\mathbf{n}(\%)\end{array}$ \\
\hline Acute myeloid leukemia (AML) & $\mathbf{n}(\%)$ & $64(47.4 \%)$ & $153(36.3 \%)$ \\
Acute lymphoid leukemia (ALL) & $186(64.8 \%)$ & $69(51.1 \%)$ & $255(60.4 \%)$ \\
Mixed phenotypic acute leukemia (MPAL) & $12(4.2 \%)$ & $2(1.5 \%)$ & $14(3.3 \%)$ \\
\hline
\end{tabular}

Table 2. Distribution of Acute Leukemias according to age group

\begin{tabular}{lccc}
\hline Types of acute leukemia & Adult $(\mathbf{n}=\mathbf{2 3 7})$ & Children $(\mathbf{n}=\mathbf{1 5 5})$ & $\begin{array}{c}\text { Total }(\mathbf{n}=\mathbf{4 2 2}) \\
\mathbf{n}(\%)\end{array}$ \\
\hline Acute myeloid leukemia (AML) & $127(83 \%)$ & $26(17 \%)$ & $153(36.3 \%)$ \\
Acute lymphoid leukemia (ALL) & $97(38 \%)$ & $158(62 \%)$ & $255(60.4 \%)$ \\
Mixed phenotypic acute leukemia (MPAL) & $13(92.90 \%)$ & $1(7.10 \%)$ & $14(3.3 \%)$ \\
\hline
\end{tabular}


$88.2 \%(75 / 85), 8.2 \%(7 / 85)$ and $3.6 \%(3 / 85)$ cases, respectively. Highest incidence of cross antigen expression was observed in T-ALL cases. Thirty four out of $41(82.9 \%)$ of T-ALLs had myeloid, B-lineage and combined myeloid-B lineage marker expression in $41.2 \%(14 / 34)$, $41.2 \% \quad(14 / 34)$ and $17.6 \% \quad(6 / 34)$ cases, respectively (Table 5, Figure 1).

\section{Discussion}

Diagnosis of acute leukemia was tradionally based on morphological and cytochemical features (FAB classification) [4], but now, flow cytometry is also one of an indispensable tool for the proper classification and diagnosis of acute leukemia.

Table 3. Distribution of AML cases according to immunophenotyping

\begin{tabular}{ll}
\hline Types of AML & $\mathbf{n = 1 5 3 ( \% )}$ \\
\hline M0 (AML without differentiation) & $10(6.7 \%)$ \\
M1 (AML without maturation) & $18(12 \%)$ \\
M2 (AML with maturation) & $47(31 \%$ \\
M3 (Acute promyelocyticleukemia, APML) & $23(15 \%)$ \\
M4 (Acute myelomonocyticleukemia) & $20(13 \%)$ \\
M5 (Acute monocyticleuekemia) & $23(15 \%)$ \\
M6 (Acute erythroblasticleukemia) & $2(1.3 \%)$ \\
M7 (acute megakaryocytic leukemia) & $6(4 \%)$ \\
t-AML (Theraly related AML) & $1(0.6 \%)$ \\
AML-MDS (AML- Myelodysplastic & $1(0.6 \%)$ \\
syndrome) & \\
\hline
\end{tabular}

Table 4. Distribution of ALL cases according to Immunophenotyping.

\begin{tabular}{lc}
\hline $\begin{array}{l}\text { Types of Acute Lymphoid } \\
\text { Leukemia (ALL) }\end{array}$ & $\mathrm{n}=255(\mathbf{1 0 0 \% )}$ \\
\hline B-ALL & $214(83.90 \%)$ \\
T-ALL & $41(16.10 \%)$ \\
\hline
\end{tabular}

Table 5. Distribution of MPAL according to immunophenotyping

\begin{tabular}{lc}
\hline Types of MPAL & $\mathbf{n}=14(100 \%)$ \\
\hline B-Myeloid & $10(71.4 \%)$ \\
T-Myeloid & $4(28.6 \%)$ \\
\hline
\end{tabular}

Table 6. Frequency of abberent Cross lineage antigen expression in acute leukemias

\begin{tabular}{lll}
\hline Leukemia & $\begin{array}{l}\text { No. of cases } \\
\mathbf{n}=\mathbf{4 0 8}\end{array}$ & $\begin{array}{l}\text { Abberant } \\
\text { cases } \mathbf{n}=159\end{array}$ \\
\hline AML & 153 & $40(26.1 \%)$ \\
B-ALL & 214 & $85(39.8 \%)$ \\
T-ALL & 41 & $34(83 \%)$ \\
\hline
\end{tabular}

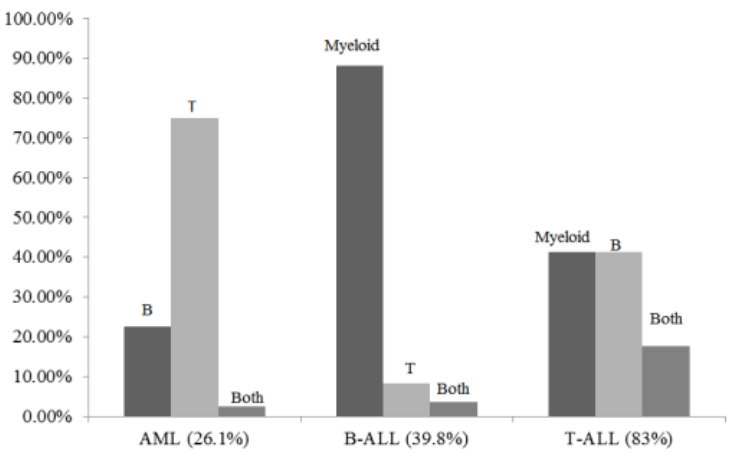

Fig. 1. Cross Lineage antigen expression in different acute leukemias.

B: B lineage cross Ag expression in AML and T-ALL.

$\mathrm{T}$ : Tlineage cross Ag expression in AML and B-ALL.

Myeloid: Myeloid lineage cross Ag expression in B-

ALL and T-ALL.

Both: Combination of B \& T, myeloid \& T and Myeloid \& B cross lineage Ag expression in AML, BALL and T-ALL, respectively.

Flow cytometry differentiate types of acute leukemia based on precursor cell expression of surface molecule that is called as cluster of differentiation (CD) antigen. Flow cytometry has also great importance in identification of biphenotypic leukemia and identification of unusual co-expression of antigen or abberent expression of CD antigen [5].

The core of monoclonal antibodies (MoAbs) investigated comprised: anti-MPO, CD13, $\mathrm{CD} 33$ and $\mathrm{CD} 117$ for the myeloid lineage cytCD3, CD2 and $\mathrm{CD} 7$ for the T-cell lineage, and CD19, CD10, CD20, and cytCD79a for the B-lymphoid lineage. All cases were also investigated for the expression of nuclear TdT, CD34, and HLA-DR and a substantial proportion for CD14, CD 64, CD 11c, CD41 and CD61.

Acute Myeloid leukemia is most common in adult whereas Acute lymphoid leukemia is common in children [6]. In our study also we observed the same frequency of acute leukemia based on age group.

In this present study, incidence of ALL was $60.4 \%$, AML was $36.3 \%$ and remaining 3.3\% was MPAL. In total AML cases, $15 \%$ cases were APML while remaining $85 \%$ were non APML. Most of the other studies also found APML ranges as $5-14 \%$, and few study stated as $23 \%$ [7]. The commonest AML subtype was AML M2 that account for $31 \%$ which is similar to the study done by Ghosh et al [8]. 
Similarly, in case of ALL B-ALL (83.90\%) is predominant to T-ALL (16.10\%). Similar study was observed in the West, the predominant immunophenotype observed in ALL was B-ALL, accounting for $60-80 \%$ of total cases, whereas TALL comprised only of $15-20 \%$ [9]. In one study from eastern India, both T-ALL (50.4\%) and BALL (49.6\%) incidence was almost equal in distribution [10].

Biphenotypic acute leukemia or MPAL is a rare type of leukemia which probably arises from hemopoietic pluripotent stem cell with the capability of differentiating along both myeloid and lymphoid ( $T$ or $B$ ) lineages of antigen expression [11]. In our study, MPAL accounted for $3.3 \%(14 / 422)$.

In present study, the aberrant cross lineage expression of myeloid antigens on lymphoid leukemias was more common than expression of myeloid antigen on lymphoid leukemias. While in other study, cross lineage antigen expression of lymphoid lineage was common in AML (12).

\section{Conclusion}

Flow cytometry is useful in correct diagnosis and subclassification of acute leukemia and is essential in cytochemical myeloperoxidase (MPO) negative cases, as well as for the diagnosis of MPALs. In present study, majority of cases were cytochemically MPO negative (including B \& T ALLs, AML-M0, M7 and MPALs). The incidence of AML and MPALs was higher in adults but ALL was predominantly seen in paediatric patients. Cross antigen lineage expression is a common phenomenon, most frequent in T-ALLs, and hence, lineage specific intra-cytoplasmic antibodies including anti-MPO and cytoplasmic-CD3 are essential for correct categorization of acute leukemias.

\section{REFERENCES}

1. Craig FE, Foon KA. Flow cytometric immunophenotyping for hematologic neoplasms. Blood. 2008; 111:3941-67.

2. Weir EG, Borowitz MJ. Flow cytometry in the diagnosis of acute leukemia, Seminar in Hematology. 2001; 38 (2): 124-38.

3. Jennings CD, Foon KA. Recent Advances in Flow Cytometry: Application to the Diagnosis of Hematologic Malignancy. Blood. 1997;90 (8): 2863-92

4. Bennett JM, Catovsky D, Daniel MT, et al. Proposals for the classification of the acute leukaemias. French-American-British (FAB) cooperative group. British Journal of Haematology. 1976; 33(4): 451-58

5. Saxena R, Anand H. Flow cytometry in acute leukemia, Renu Saxena • Hema Anand, Indian Journal of hematology and blood transfusion. 2008; 24(4):146-50
6. Sandler DP, Ross JA. Epidemiology of acute leukemia in children and adults. Seminar in Oncology. 1997; 24:3-16.

7. Salem DA, Sherin M. Flowcytometric Immunophenotypic Profile of Acute Leukemia: Mansoura Experience. Indian journal of hematology and blood transfusion. 2012 28(2), 89-96

8. Ghosh S, Shinde SC, Kumaran GS et.al. Haematologic and immunophenotypic profile of acute myeloid leukemia : an experience of Tata Memorial Hospital. Indian Journal of cancer. 2003;40 (2):71-6

9. Onciu M, Lai R, Vega F, BuesoRamos C, Medeiros LJ. Precursor Tcell acute lymphoblastic leukemia in adults: age-related immunophenotypic, cytogenetic, and molecular subsets. American Journal of Clinical Pathology. 2002;117(2):252-8
10. Mukhopadhyay A, Gangopadhyay S, gupta SD et.al. Surveillance and expected outcome of acute lymphoblastic leukemia in children and adolescents: An experience from Eastern India. Indian Journal of Medical and Paediatric Oncology. 2013;34(4):280-82.

11. Matutes E, Pickl WF, Veer MV et.al. Mixed-phenotype acute leukemia: clinical and laboratory features and outcome in 100 patients defined according to the WHO 2008 classification. Blood . 2011; 117 (11):3163-71.

12. Khurram M, Jafri SA, Mannan A et.al. Frequency of aberrant expression of $\mathrm{CD}$ marker in cases of acute leukemia. Medical Journal of Islamic World Academy of Sciences. 2010; 18 (2): 55-60. 